Western University

Scholarship@Western

Communication Sciences and Disorders

Publications

Communication Sciences and Disorders School

$1-1-2021$

\title{
Investigating the Role of Inattention and/or Hyperactivity/ impulsivity in Language and Social Functioning Using a Dimensional Approach
}

\author{
Kaitlyn M.A. Parks \\ The University of Western Ontario \\ Janis E.Oram Cardy \\ The University of Western Ontario \\ Tiffany G. Woynaroski \\ Vanderbilt University Medical Center \\ Claudia G. Sehl \\ The University of Western Ontario \\ Ryan A. Stevenson \\ The University of Western Ontario
}

Follow this and additional works at: https://ir.lib.uwo.ca/scsdpub

Part of the Communication Sciences and Disorders Commons

Citation of this paper:

Kaitlyn M.A Parks, Janis E. Oram Cardy, Tiffany G. Woynaroski, Claudia G. Sehl, Ryan A. Stevenson, Investigating the Role of Inattention and/or Hyperactivity/impulsivity in Language and Social Functioning Using a Dimensional Approach, Journal of Communication Disorders, Volume 89, 2021, 106036, https://doi.org/10.1016/j.jcomdis.2020.106036. (https://www.sciencedirect.com/science/article/pii/ S0021992420301040) 


\title{
Investigating the Role of Inattention and/or Hyperactivity/ impulsivity in Language and Social Functioning Using a Dimensional Approach
}

\author{
Kaitlyn M.A Parks ${ }^{a, b, *}$, Janis E. Oram Cardy ${ }^{\text {b,c,d }}$, Tiffany G. Woynaroski ${ }^{\text {e,f,g }}$, \\ Claudia G. Sehl ${ }^{\mathrm{a}, \mathrm{b}, \mathrm{j}}$, Ryan A. Stevenson ${ }^{\mathrm{a}, \mathrm{b}, \mathrm{d}, \mathrm{h}, \mathrm{i}}$ \\ ${ }^{\text {a }}$ Western University, Department of Psychology, 1151 Richmond St London, N6A 3K7 London, ON, Canada \\ ${ }^{\mathrm{b}}$ Western University, Brain and Mind Institute, 1151 Richmond St, London, ON, N6A 3K7, Canada \\ ${ }^{\mathrm{c}}$ Western University, School of Communication Sciences and Disorders, 1151 Richmond St, London, ON, N6A 3K7, Canada \\ ${ }^{\mathrm{d}}$ Western University, Program in Neuroscience, 1151 Richmond St, London, ON, N6A 3K7, Canada \\ ${ }^{\mathrm{e}}$ Vanderbilt University Medical Center, Department of Hearing \& Speech Sciences, 2201 West End Ave, Nashville, 377235, TN, USA \\ ${ }^{\mathrm{f}}$ Vanderbilt Kennedy Center, 2201 West End Ave, Nashville, 377235, TN, USA \\ ${ }^{\mathrm{g}}$ Vanderbilt Brain Institute, 2201 West End Ave, Nashville, 377235, TN, USA \\ ${ }^{\mathrm{h}}$ Western University, Department of Psychiatry, 1151 Richmond St, London, ON, N6A 3K7, Canada \\ ${ }^{\mathrm{i}}$ York University, Centre for Vision Research, 4700 Keele St, Toronto, ON, M3J 1P3, Canada \\ ${ }^{\mathrm{j}}$ University of Waterloo, Department of Psychology, 200 University Ave W, Waterloo, ON, N2L 3GL, Canada
}

\section{A R T I C L E I N F O}

\section{Keywords:}

Attention-deficit/hyperactivity disorder

language

social communication

social responsivity

social competency

\begin{abstract}
A B S T R A C T
The current study parsed out the distinct components of attention-deficit/hyperactivity disorder (ADHD) symptomatology to examine differential relations with language and social ability. Using a research domain criteria (RDoC) framework, we administered standardized tests and previously developed and validated questionnaires to assess levels of inattention and/or hyperactivity/ impulsivity symptomatology, language, social responsivity and social competency in 98 young adults. Those with higher inattention and/or hyperactivity/impulsivity symptomatology had reduced language comprehension, social responsivity, and social competency. Inattention and hyperactivity/impulsivity both predicted language comprehension, but not language production. Interestingly, inattention uniquely contributed to social responsiveness and social competency, but hyperactivity/impulsivity did not. Findings suggest that inattention and/or hyperactivity/ impulsivity symptoms, inattention in particular, may be especially important for social skills programs geared towards individuals with attention limitations.
\end{abstract}

\section{Introduction}

Attention-deficit/hyperactivity disorder (ADHD) is a neurobehavioral disorder characterized by inappropriate degrees of inattention and/or hyperactivity/impulsivity that significantly impact daily functioning (Association, 2013). Although language impairments are not core characteristics of $\mathrm{ADHD}$, frequent co-occurrence of these difficulties has been well-documented in the ADHD

\footnotetext{
* Corresponding author at: Western Interdisciplinary Research Building, 1151 Richmond St, London, ON, N6A 3K7, Canada.

E-mail addresses: kparks7@uwo.ca (K.M.A Parks), janis.cardy@uwo.ca (J.E.O. Cardy), tiffany.g.woynaroski@vumc.org (T.G. Woynaroski), c2sehl@uwaterloo.ca (C.G. Sehl),rsteve28@uwo.ca (R.A. Stevenson).
} 
literature (Geurts \& Embrechts, 2008; Gremillion \& Martel, 2014; Korrel, Mueller, Silk, Anderson, \& Sciberras, 2017; Mueller \& Tomblin, 2012; Purvis \& Tannock, 1997; Redmond, Ash, \& Hogan, 2015; Redmond, 2016). Estimates have suggested that upwards of 40-60\% of children with ADHD present with comorbid language impairments (Bruce, Thernlund, \& Nettelbladt, 2006; Cohen, Davine, Horodezky, Lipsett, \& Isaacson, 1993; Hagberg, Miniscalco, \& Gillberg, 2010; Oram, Fine, Okamoto, \& Tannock, 1999; Sciberras et al., 2014).

Individuals with ADHD with comorbid language impairment have been described as experiencing a variety of language and communication difficulties. Such difficulties can include impairments in acquisition and functional use of expressive, receptive, and pragmatic language relative to typically developing peers (Geurts \& Embrechts, 2008; Green, Johnson, \& Bretherton, 2014; Korrel et al., 2017; Purvis \& Tannock, 1997; Staikova, Gomes, Tartter, McCabe, \& Halperin, 2013). Difficulties related to pragmatic language are commonly reported (Staikova et al., 2013), and can include excessive talking, failure to understand verbal and non-verbal cues, poor turn-taking during conversation, difficulty maintaining appropriate physical proximity, and poorly organized expressive language (Green et al., 2014). It is possible that, even in the absence of a co-occurring developmental language disorder (DLD), core behavioral symptoms of ADHD such as distractibility and/or impulsivity may lead to important linguistic information or social cues being missed, misinterpreted, or forgotten, resulting in reduced learning of elements that could enhance language and social functioning (Oram et al., 1999).

Language and social difficulties in individuals with increased levels of inattention and/or hyperactivity/impulsivity are present early in life, with studies showing that increased ADHD characteristics in children as young at four years old are linked with language difficulties (Geurts \& Embrechts, 2008). In particular, language difficulties related to pragmatic and structural language difficulties have been associated with the severity of symptoms in inattention and/or hyperactivity/impulsivity (Hawkins, Gathercole, Astle, Holmes, \& Team, 2016) and can include excessive talking, difficulties maintaining appropriate conversational rapport, and failure to respond to verbal cues. Deficits related to components of structural language including syntax and phonology have also been documented in individuals as young as three and as old as 18 years of age (Green et al., 2014). Impairments related to broader social skills have been reported as early as the preschool years by parents, teachers, and peers (DuPaul, McGoey, Eckert, \& VanBrakle, 2001). Such social challenges can lead to increased peer rejection (Redmond, 2011) and academic failure early on in life (Barkley, 1997; Wilens \& Spencer, 2010), and extend into adulthood to impact vocational outcomes (Friedman et al., 2003; Russell, Jarrold, \& Hood, 1999). In turn, peer rejection and academic failure may lead to an increased avoidance of social interactions, and without this engagement, pre-existing social and language difficulties are likely to worsen (Redmond, 2011).

One possible explanation for poor language and communication skills is that difficulties related to attention and hyperactivity/ impulsivity may disrupt the ability to learn successful language and social skills, at least through formal methods of teaching. Previous work has demonstrated that inattention and hyperactivity/impulsivity symptoms can produce different patterns of behavior (Barkley, DuPaul, \& McMurray, 1990; Martel, Von Eye, \& Nigg, 2010; Toplak et al., 2009) and therefore have the potential to differentially impact language and social outcomes. For instance, several studies have demonstrated that individuals with ADHD tend to have reduced expressive, but not receptive language abilities (Barkley et al., 1990; Kim \& Kaiser, 2000; Munir, Biederman, \& Knee, 1987). Other researchers have reported that individuals with attention deficits show reduced receptive and expressive language abilities (Baker \& Cantwell, 1992) while those with increased impulsivity show greater deficits related to structural language (Geurts \& Embrechts, 2008). Further, individuals with increased inattention display deficits related to social knowledge (Wheeler Maedgen \& Carlson, 2000), social passivity, low acceptance by peers, and increased aggression (Bellanti \& Bierman, 2000) while those with combined inattention and hyperactivity/impulsivity display higher levels of aggressive behavior, high levels of both positive and negative behavior, and emotional dysregulation (Wheeler Maedgen \& Carlson, 2000). It is not surprising that inattention and/or hyperactivity/impulsivity is associated with poor language and social skills given that successful communication depends on the ability to maintain attention, focus on others, and follow the rules of nonverbal interaction, areas which represent core challenges in ADHD (Bruce et al., 2006). It is therefore of interest to examine the associations between language, social abilities, and inattention and hyperactivity/impulsivity in order to better understand the language and social profiles of these individuals.

Clinical definitions of ADHD may fall short in capturing some of the less pronounced, milder symptoms associated with the disorder (Cuthbert, 2014; Insel et al., 2010). It is important to move beyond evaluating skills and behaviors as either impaired or unimpaired when assessing mental disorders (Cuthbert, 2014; Insel et al., 2010). That is, an individual may demonstrate milder symptoms associated with a disorder where such symptoms do not reach clinical significance. However, these behaviors may have a direct and negative impact on their social, occupational, and academic success. In order to improve upon diagnostic precision, it is important to first understand how specific behaviors associated with a disorder relate to other behaviors that can be observed in the general population. The National Institute of Mental Health (NIMH) has initiated the RDoC framework as one way to accomplish this goal (NIMH, 2008). Rather than using traditional diagnostic categories, the framework encourages researchers to consider other units of analysis that can cut across disorders as the starting point for grouping individuals with mental disorders (Cuthbert \& Insel, 2013; Insel et al., 2010). For example, researchers can combine behavioural paradigms, self-reports, and physiological measures such as electroencephalography and functional magnetic resonance imaging to investigate patterns of dysfunction independent of traditional diagnoses. The framework is not intended to act as a diagnostic guide or replace current diagnostic systems. Rather, the approach encourages researchers to investigate different disorders in terms of varying degrees of dysfunction. The framework contains five constructs that can be assessed including cognition, negative and positive valence systems, social process systems, and arousal/modulatory systems, each of which can be subjected to multiple levels of analysis that include genes, cells, physiology, neural circuits, and behavior. The RDoC approach can therefore be used to examine the entire spectrum of symptoms, from clearly disordered to subclinical to within the normal range.

Previous work has demonstrated that subclinical levels of inattention and hyperactivity/impulsivity are present in the general 
population (Das, Cherbuin, Butterworth, Anstey, \& Easteal, 2012) and that inattention and/or hyperactivity/impulsivity symptoms are rather common, with $60 \%$ of individuals displaying at least one symptom of hyperactivity or inattention (Arcos-Burgos \& Acosta, 2007). Further, research has shown that there is large variability in ADHD prevalence rates (Polanczyk, De Lima, Horta, Biederman, \& Rohde, 2007). For example, within a single geographic location, prevalence rates of ADHD varied from $3.7 \%$ to $8.9 \%$ depending on the specific methodological criteria used such as the presence or absence of impairment (Polanczyk et al., 2007). The RDoC framework can inform future classifications by integrating various levels of information including genes, molecules, cells, circuits, physiology, behavior, and self-reports, rather than descriptive phenomenology and categorical definitions alone. Indeed, application of the RDoC framework has provided information to support previously developed new subgroups based on their attention characteristics that do not align with original diagnostic classifications (Kleinman et al., 2015). In this study, children with diagnosed ADHD, bipolar disorder (BD), and ADHD + BD were clustered based on their patterns of attention, independent of original diagnoses. Researchers found that patterns of attention were best described through two new subgroups, each of which contained a combination of ADHD, BD, and ADHD $+\mathrm{BD}$ children. This framework therefore has the potential to advance us towards our long-term objective of developing novel approaches to intervention for those experiencing varying levels of a disorder. In the current study, we followed the RDoC approach by examining the dimensions of inattention and hyperactivity/impulsivity in a sample of young adults who were not required to have a formal ADHD diagnosis.

Through this approach, we aimed to better understand the relations between inattention and/or hyperactivity/impulsivity, and social and language abilities. First, we hypothesized that inattention and/or hyperactivity/impulsivity would be associated with lower language and social abilities. Second, given that inattention and hyperactivity/impulsivity are characterized as distinct behavioral symptoms in ADHD (Barkley et al., 1990; Martel et al., 2010; Toplak et al., 2009) and have been shown to differentially impact language (Baker \& Cantwell, 1992; Geurts \& Embrechts, 2008) and social functioning (Wheeler Maedgen \& Carlson, 2000), we predicted that levels of hyperactivity/impulsivity and inattention would have qualitatively different effects on language and social functioning. No specific predictions regarding the nature of these contributions were made as research in this area has reported mixed findings. In the current study, we tested these two hypotheses by administering a psychometrically sound measure of language comprehension and production with the prediction that higher inattention and/or hyperactivity/impulsivity would relate to lower language skills. Further, we assessed whether higher inattention and/or hyperactivity/impulsivity was related to social skill by administering two previously developed and validated measures of social responsiveness and social competence. We examined the inattention and hyperactivity/impulsivity components of an ADHD scale to determine whether either or both of these uniquely contribute to language and social functioning.

\section{Method}

\subsection{Participants}

An initial sample of 104 young adults were recruited for the current study. Six participants were excluded from further analysis, five for being over the maximum age for which the standardized language measure intended, and one for failure to complete the protocol. Only participants who were English speakers and had self-reported normal or corrected-to-normal vision and normal hearing were included in the analysis. Participants were recruited from the undergraduate psychology pool at the University of Western Ontario as part of a larger study on developmental disabilities and statistical learning (Parks \& Stevenson, 2018). In the current study, we followed an RDoC approach and therefore, participants were not required to have a formal ADHD diagnosis. This resulted in a final sample of 98 participants, four of which had reported a diagnosis of ADHD. Ages ranged from 16 to 21 years (Mean age =18.19 \pm .74 years), and $63(64.3 \%)$ participants were female.

\subsection{Procedures}

Participants completed a battery of tests, including a standardized clinical measure of receptive and expressive language, and selfreport questionnaires examining social responsiveness, social competency, and inattention and/or hyperactivity/impulsivity symptomatology. These tasks took approximately one hour to complete, and all protocols were approved by the University of Western Ontario Research Ethics Board.

\subsubsection{Standardized language assessment}

Receptive and expressive language abilities were measured by administering six subtests from the Clinical Evaluation of Language Fundamentals, Fifth Edition (CELF-5; Wiig, Semel, Secord, \& Pearson Education, 2013): Word Classes, Understanding Spoken Paragraphs, Semantic Relationships, Formulated Sentences, Recalling Sentences, and Sentence Assembly. Receptive and expressive language standard scores were used as metrics of receptive and expressive language ability, respectively, in analyses.

\subsubsection{Standardized measure of inattention and/or hyperactivity/impulsivity}

The Adult ADHD Self-Report Scale (ASRS-v1.1; Kessler et al., 2005) was used to measure inattention and/or hyperactivity/impulsivity symptoms. The ASRS was chosen for the current study because is a reliable screening measure that has been shown to be successful in screening for ADHD characteristics in the general population (Kessler et al., 2005, 2007) and is therefore well-suited for the RDoC approach used in the current study. On this 18-item measure, participants respond on a five-point Likert scale (never, rarely, sometimes, often, or very often) to reflect the frequency with which they experience challenges with attention and/or 
hyperactivity/impulsivity. Each question is followed by 5 response options to capture symptom severity (i.e., never, rarely, sometimes, often, and very often) where responses of 'rarely' or 'never' were scored as ' 0 ' while responses of 'sometimes', 'often', and 'very often' were scored as ' 1 ',

Inattention and/or hyperactivity/impulsivity symptomatology was quantified by scoring responses for each of the 18 items of the ASRS as " 0 " or " 1 " in accordance with Kessler et al. (2005) to derive a total score (sum of scores across all 18 items), as well as scores for inattention (sum of items tapping inattention) and hyperactivity/impulsivity (sum of scores tapping hyperactivity/impulsivity). Inattention is measured by nine questions on the ASRS, for example, "How often do you have trouble wrapping up the fine details of a project, once the challenging parts have been done?" Hyperactivity/impulsivity is measured by an additional nine items on the ASRS, for example, "How often do you feel overly active and compelled to do things, like you were driven by a motor?"

Measures of social skill. Social skill was measured via the Social Responsiveness Scale (SRS; Constantino et al., 2003) and the Multidimensional Social Competence Scale (MSCS; Yager \& Iarocci, 2013). The SRS is a 65-item measure that evaluates a range of social difficulties and serves as an index for severity of social deficits. Participants are instructed to respond using a four-point Likert scale (not true; sometimes true; often true; almost always true) to statements indexing social awareness, social communication, and social cognition. Scores can range from 0 to 195 with those that are higher representing greater social impairment. Example items on this scale include "I behave in ways that seem strange or bizzare to others" and "I am awkward in turn-taking interactions with others". The MSCS is a 77-item measure that evaluates an individuals ability to adequately respond and adjust to social demands with a specific focus on the relational aspects of social interactions between individuals. The measure is useful in comparing patterns of social competence across both clinical and non-clinical populations. Participants are instructed to respond using a five-point Likert scale (not true/almost never true; rarely true; sometimes true; often true; very true/almost always true) to statements on social motivation, inferencing, knowledge, empathy, verbal and non-verbal skills, and emotion regulation. Scores can range from 0 to 385 with higher scores indicating greater agreement and greater social competence. Example items include "I am sensitive to the feelings and concerns of others" and "I misread social cues".

Responses on the SRS and MSCS were calculated in accordance with the manuals for each measure. The SRS includes two indices of social communication: Social Communication and Interaction (SCI) and Restricted and Repetitive Behavior (RRB). Scores on the RRB subscale of the SRS were subtracted from total t-scores in the current study, as outlined by the manual, to ensure that the measure was not tapping into other clinical symptomatology. Thus, SCI and not RRB scores were considered in our analysis. Participants' social responsiveness score was now reflective of their social communication index score, measuring only Social Communication and Interaction. For both measures, total scores were used in subsequent analyses.

\subsection{Analysis}

All variables used in analyses were found to be normally distributed and therefore, bivariate correlations were used to examine relationships between total $\mathrm{ADHD}$, language, and social skills with a Benjamini-Hochberg false discovery rate procedure $(\mathrm{Q}=.1)$ used to correct for multiple comparisons. Linear regressions were carried out to examine whether the distinct components of ADHD, inattention and hyperactivity/impulsivity, uniquely predicted language and social abilities. For all regression analyses, inattention and hyperactivity/impulsivity sub scores were included as predictors.

\section{Results}

\subsection{Adult ADHD Self-Report Scale}

Scores on the ASRS spanned nearly the entire scale of inattention and/or hyperactivity/impulsivity symptomatology (with a range of $0-17$ out of a possible 18 points on the ASRS). Previous standardization of the ASRS identified the cut point as between 10 and 11 as optimal in predicting clinical-level symptoms with a classification accuracy of $96.2 \%$ (specificity $=98.3 \%$, sensitivity $56.3 \%$ ) (Kessler et al., 2007). The current sample included 16 participants (16.33\%) scoring at levels considered at-risk for ADHD, with those reporting an ADHD diagnosis having total inattention and/or hyperactivity scores ranging from 5 to 15. One participant who reported an ADHD diagnosis scored within the normal range, while the rest had total scores above the cut point for clinical symptomatology. Please see Table 1 for descriptive statistics for all variables of interest.

Table 1

Descriptive Statistics and Ranges for Variables of Interest.

\begin{tabular}{llll}
\hline & Mean & S.D & Range \\
\hline 1. Total ADHD & 6.51 & 4.02 & $0-17$ \\
2. Inattention & 3.86 & 2.47 & $0-9$ \\
3. Hyperactivity/Impulsivity & 2.65 & 2.13 & 0.32 \\
4. Receptive Language & 98.72 & 8.36 & $75-120$ \\
5. Expressive Language & 101.50 & 10.09 & $48-120$ \\
6. Social Responsiveness & 60.11 & 30.46 & $194-960$ \\
7. Social Competency & 292.07 & & \\
\hline
\end{tabular}

Note. S.D indicates standard deviation. 


\subsection{Relating Total ADHD, Language, and Social Abilities}

Total inattention and/or hyperactivity/impulsivity was significantly related to receptive language abilities $\left(r{ }_{(97)}=-.30, \mathrm{CI}_{95 \%}=\right.$ -.47 to $-.11, p=.003$ ) indicating that higher inattention and/or hyperactivity/impulsivity was associated with lower receptive language abilities (Fig. 1). Expressive language, on the other hand, was not significantly related to total inattention and/or hyperactivity/ impulsivity $\left(r(97)=-.13, p=.202, \mathrm{CI}_{95 \%}=-.32\right.$ to .07). Further, total SRS and MSCS scores were significantly related to total inattention and/or hyperactivity/impulsivity symptomatology $\left(r_{(97)}=.51, p<.001, \mathrm{CI}_{95 \%}=.35\right.$ to .64 and $r(97)=-.42, p<.001, \mathrm{CI}_{95 \%}=$ -.57 to -.24 , respectively) indicating that higher inattention and/or hyperactivity/impulsivity symptomatology was associated with greater impairments in social responsivity and lower social competency (Fig. 2).

\subsection{Regression Analyses}

To determine whether inattention and hyperactivity/impulsivity uniquely contributed to receptive language abilities, a multiple regression was performed. The overall model was found to be significant, $F_{(2,95)}=4.58, p=.013, \mathrm{R}^{2}=.09, \mathrm{f}^{2}=.10$. However, neither inattention $\left(\beta=-.83, t{ }_{(95)}=-1.73, \mathrm{CI}_{95 \%}=-1.78\right.$ to $.13, p=.088, s r^{2}=0.029 ; r_{(97)}=-.27, p=.007, \mathrm{CI}_{95 \%}=-.44$ to -.08$)$ nor hyperactivity/impulsivity $\left(\beta=-.68, t(95)=-1.21, \mathrm{CI}_{95 \%}=-1.79\right.$ to $.43, p=.228, s r^{2}=0.014 ; r_{(97)}=-.24, p=.016, \mathrm{CI}_{95 \%}=-.42$ to -.04$)$ reached statistical significance in this model (Fig. 3). A second multiple regression was performed to determine whether inattention and hyperactivity/impulsivity uniquely contributed to expressive language abilities. The overall model was not significant, $F(2,95)=$ $1.42, p=.246, \mathrm{R}^{2}=.03, \mathrm{f}^{2}=.03$. Likewise, neither inattention $\left(\beta=-.64, t_{(95)}=-1.60, \mathrm{CI}_{95 \%}=-1.44\right.$ to $.15, p=.112, s r^{2}=0.026 ; r_{(97)}$ $=-.17, p=.103, \mathrm{CI}_{95 \%}=-.36$ to .03$)$ nor hyperactivity/impulsivity $\left(\beta=.19, t_{(95)}=.40, \mathrm{CI}_{95 \%}=-.74\right.$ to $1.11, p=.693, s r^{2}=0.002 ; r$ (97) $=-.05, p=.606, \mathrm{CI}_{95 \%}=-.25$ to .15 ) were found to be statistically significant in this model.

To assess whether inattention and hyperactivity/impulsivity uniquely contributed to social responsiveness, an additional multiple regression was performed, and the overall model was found to be significant, $F_{(2,95)}=17.17, p<.001, \mathrm{R}^{2}=.27, \mathrm{f}^{2}=.37$. Further, inattention $\left(\beta=1.67, t{ }_{(95)}=3.96, \mathrm{CI}_{95 \%}=.83\right.$ to $2.51, p<.001, s r^{2}=0.121 ; r_{(97)}=.50, p<.001, \mathrm{CI}_{95 \%}=.34$ to .64$)$ but not hyperactivity/impulsivity $\left(\beta=.787, t_{(95)}=1.61, \mathrm{CI}_{95 \%}=-.19\right.$ to $1.76, p=.112, s r^{2}=0.020 ; r_{(97)}=.38, p<.001, \mathrm{CI}_{95 \%}=.20$ to .54$)$ was found to be statistically significant in this model (Fig. 4). A final multiple regression was performed to determine whether inattention and hyperactivity/impulsivity uniquely contributed to individual differences in social competency, and the overall model was significant, $F_{(2,95)}=10.80, p=<.001, \mathrm{R}^{2}=.19, \mathrm{f}^{2}=.23$. A similar pattern emerged where inattention $\left(\beta=-4.14, t_{(95)}=-3.09, \mathrm{CI}_{95 \%}=\right.$ -6.80 to $-1.48, p=.003, s r^{2}=0.082 ; r_{(97)}=-.41, p<.001, \mathrm{CI}_{95 \%}=-.56$ to -.23$)$, but not hyperactivity/impulsivity $(\beta=-2.09, t(95)=$ $-1.34, \mathrm{CI}_{95 \%}=-5.18$ to $1.01, p=.184, s r^{2}=0.015 ; r_{(97)}=-.32, p=<.01, \mathrm{CI}_{95 \%}=-.49$ to -.13 ) was found to be statistically significant in this model. Please see Table 2 for bivariate correlations between ADHD symptoms, language, and social Skills

\section{Discussion}

The current study examined how overall inattention and/or hyperactivity/impulsivity symptomatology as well as inattention and hyperactivity/impulsivity uniquely relate to individual differences in language and social difficulties. Our data support the hypothesis that those with higher subclinical levels of inattention and/or hyperactivity/impulsivity tend to have lower receptive language and social abilities. Both inattention and hyperactivity/impulsivity were related to lower language comprehension. However, only inattention uniquely contributed to social responsiveness and competency abilities in our sample. These results have the potential to inform future studies that wish to examine the broad constructs of inattention and/or hyperactivity/impulsivity and how these relate to language and social functioning in different populations. Such studies could inform interventions targeting ADHD symptomatology which may have the potential to translate to effects on those with clinical levels of ADHD as well as higher-level language and social skills, and vice versa.

Surprisingly, we did not find support for a relationship between inattention and/or hyperactivity/impulsivity symptomatology and expressive language abilities, despite various studies finding a link between the two (Cardy, Tannock, Johnson, \& Johnson, 2010; Gut, Heckmann, Meyer, Schmid, \& Grob, 2012; Hutchinson, Bavin, Efron, \& Sciberras, 2012; Jonsdottir, Bouma, Sergeant, \& Scherder, 2005; Martinussen \& Tannock, 2006; McInnes, Humphries, Hogg-Johnson, \& Tannock, 2003) with a number using the same language
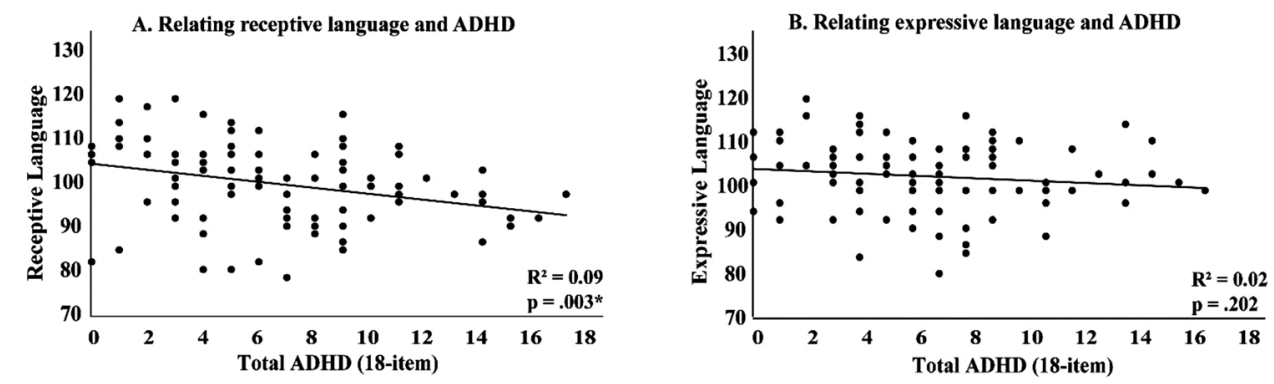

Fig. 1. Relating receptive language and total ADHD (A) and expressive language and total ADHD (B). 

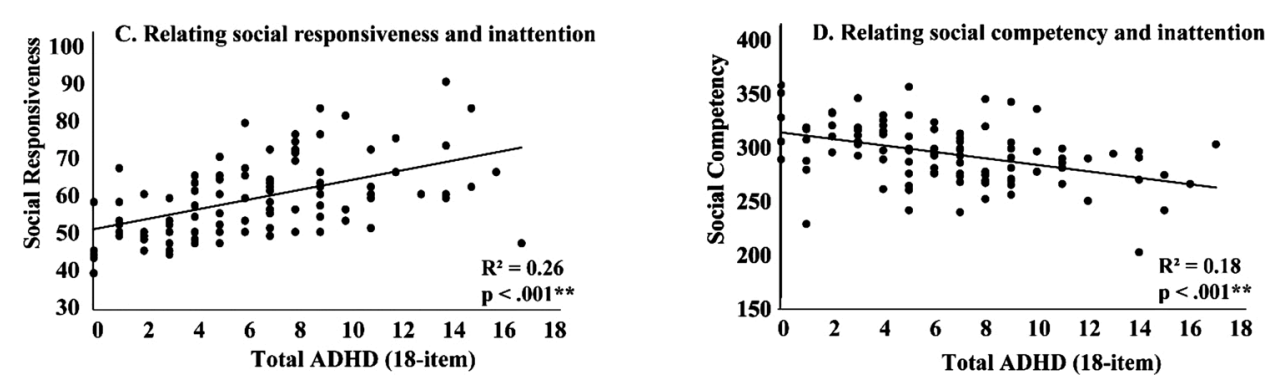

Fig. 2. Relating social responsiveness and total ADHD (C) and social competency and total ADHD (D).
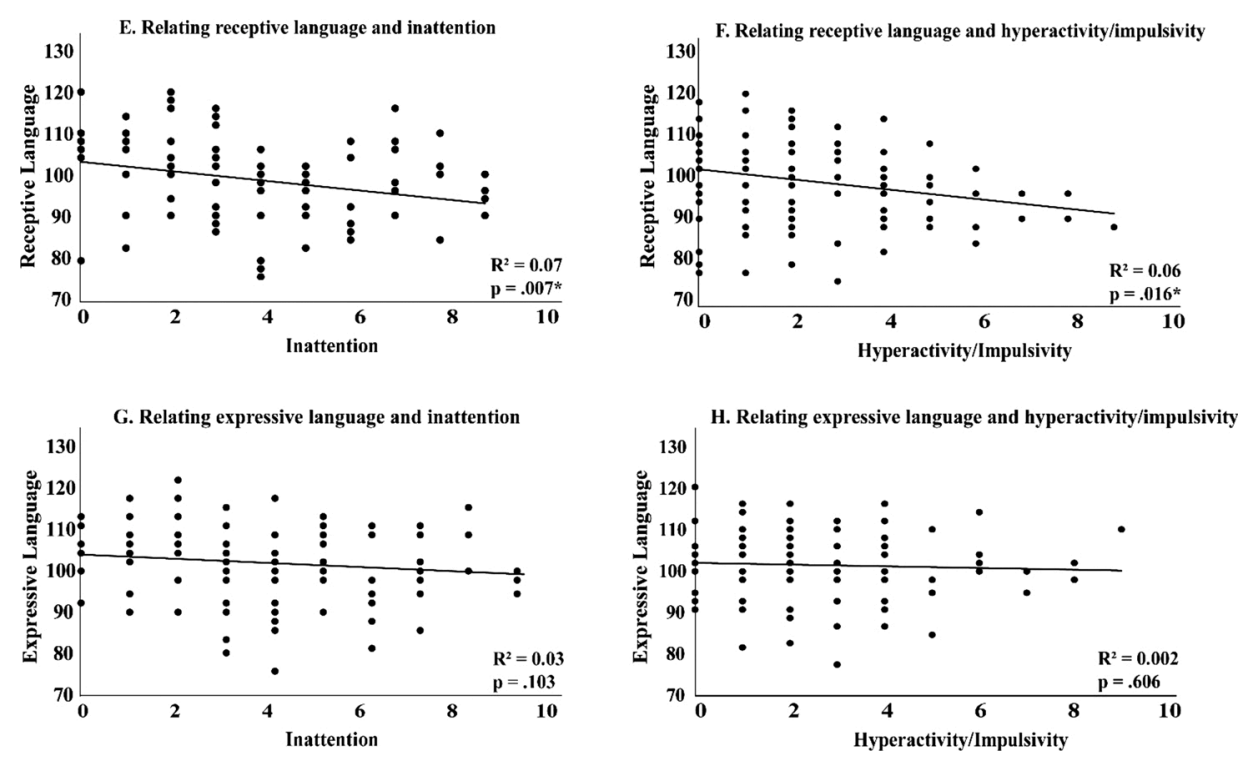

Fig. 3. Relating receptive language and inattention (E), receptive language and hyperactivity/impulsivity (F), expressive language and inattention $(\mathrm{G})$, and expressive language and hyperactivity/impulsivity $(\mathrm{H})$.
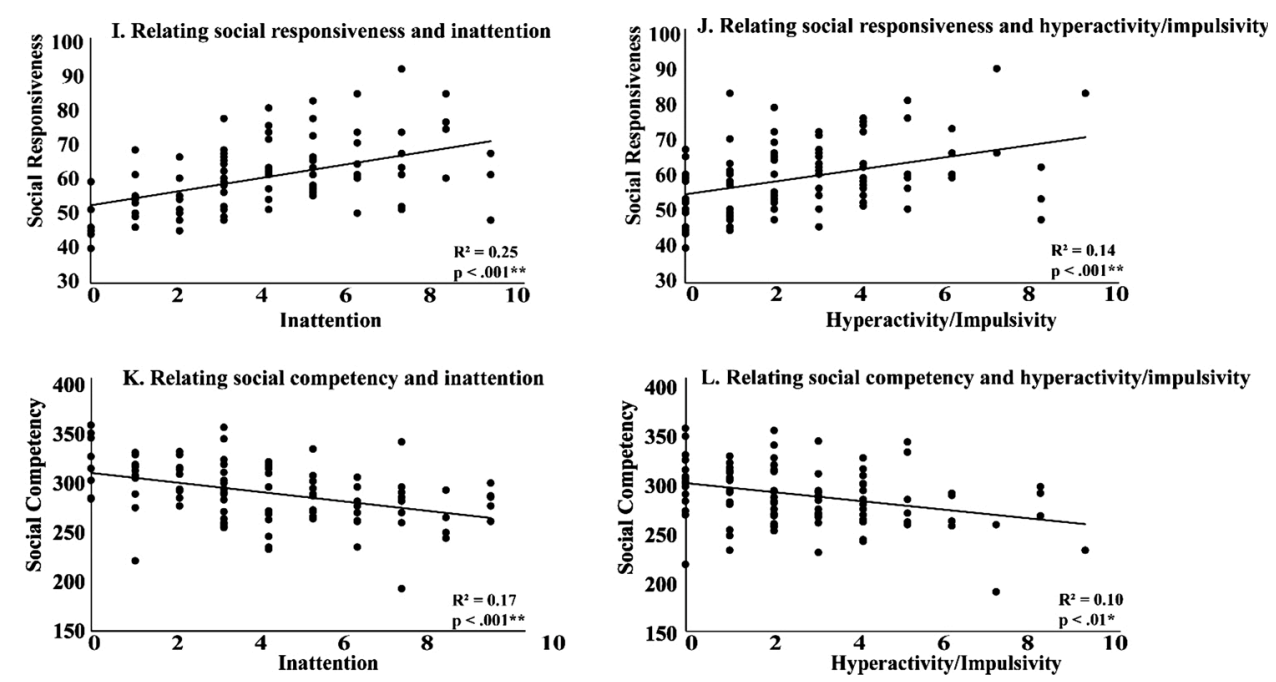

Fig. 4. Relating social responsiveness and inattention (I), social responsiveness and hyperactivity/impulsivity (J), social competency and inattention (K), and social competency and hyperactivity/impulsivity (L). 
Table 2

Bivariate correlations between ADHD Symptoms, Language, and Social Skills (N = 98).

\begin{tabular}{|c|c|c|c|c|c|c|c|}
\hline & 1 & 2 & 3 & 4 & 5 & 6 & 7 \\
\hline 1. Total ADHD & - & - & - & - & - & - & - \\
\hline 2. Inattention & $.89^{* *}$ & - & - & - & - & - & - \\
\hline 3. Hyperactivity/Impulsivity & $.85^{* *}$ & $.52 * *$ & - & - & - & - & - \\
\hline 4. Receptive Language & $-.30 * *$ & $-.27 * *$ & $-.24 *$ & - & - & - & - \\
\hline 5. Expressive Language & -.13 & -.17 & -.05 & $.51^{* *}$ & - & - & - \\
\hline 6. Social Responsiveness & $.51^{* *}$ & $.50 * *$ & $.38^{* *}$ & -.17 & -.07 & - & - \\
\hline 7. Social Competency & $-.42 * *$ & $-.41 * *$ & $-.32 * *$ & $.22 *$ & $.21 *$ & $-.80 * *$ & - \\
\hline
\end{tabular}

Note. ${ }^{*} \mathrm{p}<.05 ; * * \mathrm{p}<.01$.

measure that was used in the current study (Gut et al., 2012; Martinussen \& Tannock, 2006; McInnes et al., 2003). However, most studies finding a link between expressive language and ADHD have used child participants with a formal diagnosis of ADHD. Thus, it could be that difficulties related to expressive language are more salient in younger groups with a clinical diagnosis. Our participants are quite different from those of comparative studies that have linked expressive language and inattention and/or hyperactivity/impulsivity symptomatology in clinical samples, and it should therefore be noted that comparable findings may have emerged if we included those with a clinical diagnosis of ADHD. Studies have found that individuals with ADHD tend to have difficulties in areas related to expressive language but not receptive language (Barkley et al., 1990; Munir et al., 1987) while others have shown that both areas of language are impaired (Baker \& Cantwell, 1992). It is difficult to compare or generalize such findings to those of the current study due to age and methodological differences. Prior work has noted that linguistic tasks used to measure receptive and expressive language tend to differ across studies and few studies examining language outcomes in ADHD have included comparisons groups (Kim \& Kaiser, 2000). Thus, the differential associations found between inattention and/or hyperactivity/impulsivity and receptive and expressive language in the current study could be, in part due to these distinct methodological differences.

Our findings support the hypothesis that language comprehension and social functioning are associated with inattention and/or hyperactivity/impulsivity symptomatology (e.g. Geurts \& Embrechts, 2008; Green et al., 2014; Korrel et al., 2017; Purvis \& Tannock, 1997; Staikova et al., 2013), using an RDoC approach. Research suggests that individuals with high levels of inattention and hyperactivity/impulsivity are less able to establish and maintain conversational rapport (Bishop \& Baird, 2001) and are more likely to take shortcuts to achieve a communicative goal (Leonard, Milich, \& Lorch, 2011) - tendencies which have clear potential to impact social success.

One novel finding of the current study was that inattention uniquely and significantly contributed to social responsiveness and social competency, but hyperactivity/impulsivity did not. Previous research has shown that these two characteristics of ADHD are qualitatively different from one another and can produce different patterns of behavior (Barkley et al., 1990; Martel et al., 2010; Toplak et al., 2009) - those with solely a tendency towards inattention are described as more confused, lost in thought, and apathetic than those with both inattention and hyperactivity/impulsivity. Additional research has found that inattention in ADHD contributes to both social behavior and peer relationships, with higher degrees of inattention being predictive of deficits in social skills, elevated levels of aggression and disruptive behavior, and high ratings of dislike by peers (Bellanti \& Bierman, 2000). Given that attention is an important component of social functioning, it is perhaps no surprise that skills related to social responsiveness and social competency, including social motivation and verbal conversation skills, are negatively impacted by reduced levels of attention. That is, in order to learn the rules or conventions governing language and social interactions, individuals must first properly allocate their attentional resources by focusing on important details and overlooking irrelevant ones. An inability to actively maintain focus in the environment may lead to important information concerning language and social functioning being either neglected or misinterpreted.

Importantly, we have shown here that an RDoC framework can be used to successfully capture the language and social difficulties in those without a formal diagnosis of ADHD, but a distribution of inattention and/or hyperactivity/impulsivity symptoms. Gathering information about the associations between language and social functioning through this framework encourages researchers to investigate these associations in larger, more diverse samples who do not have a formal diagnosis, leading to increases in statistical power and better classifications based on a range of symptomatology.

\section{Future Directions and Limitations}

There are several important considerations for future work that should be noted. First, although we did not have a clinically diagnosed sample of young adults with ADHD, it is important for future work to consider that some tasks (e.g., certain standardized language tasks) can place high demands on aspects of cognitive functioning that are impaired in ADHD including attention, planning, and inhibition, making these tasks especially challenging for these individuals (Oram et al., 1999). Evaluating how aspects of executive function may be contributing to findings for between-group differences, as well as observed associations with language, social functioning, and other skills thus represents an important direction for future research.

Second, future work should compare other groups who present with language and social difficulties overlapping with those seen in individuals with ADHD such as those with DLD and autism spectrum disorder (ASD) using an RDoC approach. Specific abilities that should be compared include those examined here, receptive and expressive language, as well as social responsiveness and social competence. This method would enable researchers to evaluate language and social functioning across related diagnoses to understand the degrees of convergence and divergence occurring across diagnostic groups. One example of why this approach might be important 
is that some individuals are diagnosed with ADHD and co-occurring DLD. These individuals may present with different behavioral profiles than those with ADHD alone and those with DLD alone but may receive a combination of interventions targeted towards individuals who present with the disorders in isolation. Ascertaining the overlap and non-overlap of symptoms may inform our development of more precisely targeted treatment packages and advance us towards the achievement of more optimal outcomes across clinical populations known to experience challenges in attention, language, socialization, and other domains.

Finally, it is important to note that although the questionnaires used in the current study were intended to measure inattention and/ or hyperactivity/impulsivity, social responsiveness, and social competency as separate components, it is possible that each measure is assessing a similar, underlying construct and that certain items may be tapping into additional clinical symptomatology. For example, some items on the ASRS may be tapping into issues related to social function, in addition to attention. Further, the SRS and MSCS have been used in previous work focused on social function in ASD (Bölte, Poustka, \& Constantino, 2008; Yager \& Iarocci, 2013), Tuberous sclerosis complex (Granader et al., 2010), and epilepsy (Viscidi et al., 2014), indicating that although these measures are reliable in assessing social function across a range of diagnostic groups the total scores may not simply be a reflection of social function and rather social issues that can arise as a function of these disorders. Thus, while we believe the ASRS to be a valid and reliable measure of inattention and/or hyperactivity/impulsivity symptomatology, it is possible that additional issues related to social function are being evaluated through this measure.

\section{Conclusions}

Taken together, the results from the current study add to the growing literature suggesting associations between inattention and/or hyperactivity/impulsivity symptoms, language, and social skills. Our results provide novel evidence to suggest that (a) inattention and/or hyperactivity/impulsivity symptoms may contribute more so to difficulties with language comprehension than language production, and (b) that inattention may contribute more so to reduced social responsiveness and social competency than hyperactivity/impulsivity. Therefore, behavioral symptoms related to inattention may be an important focus of social skills training for individuals who experience deficits in attention, whereas ADHD traits more broadly speaking may be important targets for individuals with inattention and/or hyperactivity/impulsivity who struggle to understand language. This study is the first to evaluate the differential associations between inattention and hyperactivity/impulsivity to language and social abilities in a sample with largely subclinical levels of inattention and/or hyperactivity/impulsivity symptomatology. Further, we have shown here that reduced language and social skills are not restricted to those with diagnosed ADHD but are represented in the general population as well. Thus, our results suggest that at least some core symptoms of ADHD can be examined in the general population to inform the development of novel interventions and allow researchers to administer tasks that might otherwise be too attentionally demanding for those diagnosed with ADHD.

\section{Funding}

This work was funded by grants to RAS by an NSERC Discovery Grant (RGPIN-2017-04656), a SSHRC Insight Grant (435-20170936), the University of Western Ontario Faculty Development Research Fund, and the John R. Evans Leaders Fund from the Canadian Foundation for Innovation (Project \#37497), and to KMAP by the Western Graduate Research Scholarship, and Ontario Graduate Scholarship, the Kingsley Allison Research Award, and the Autism Scholars Award. The above funding sources had no involvement in the study design, data collection, analysis and interpretation of data, the writing of the report, and in the decision to submit the article for publication.

\section{CRediT authorship contribution statement}

Kaitlyn M.A Parks: Conceptualization, Methodology, Formal analysis, Writing - original draft. Janis E. Oram Cardy: Writing review \& editing. Tiffany G. Woynaroski: Writing - review \& editing. Claudia G. Sehl: Investigation, Resources. Ryan A. Stevenson: Writing - review \& editing, Supervision, Funding acquisition.

\section{References}

Arcos-Burgos, M., \& Acosta, M. T. (2007). Tuning major gene variants conditioning human behavior: the anachronism of ADHD. Current opinion in genetics \& development, 17(3), 234-238.

Association, A. P. (2013). Diagnostic and statistical manual of mental disorders (5ta. ed.). Washington, DC: American Psychiatric Association.

Baker, L., \& Cantwell, D. P. (1992). Attention deficit disorder and speech/language disorders. Comprehensive mental health care.

Barkley, R. A. (1997). Behavioral inhibition, sustained attention, and executive functions: constructing a unifying theory of ADHD. Psychological bulletin, 121(1), 65-94.

Barkley, R. A., DuPaul, G. J., \& McMurray, M. B. (1990). Comprehensive evaluation of attention deficit disorder with and without hyperactivity as defined by research criteria. Journal of consulting and clinical psychology, 58(6), 775.

Bellanti, C. J., \& Bierman, K. L. (2000). Disentangling the impact of low cognitive ability and inattention on social behavior and peer relationships. Journal of Clinical Child Psychology, 29(1), 66-75.

Bishop, D. V., \& Baird, G. (2001). Parent and teacher report of pragmatic aspects of communication: use of the Children's Communication Checklist in a clinical setting. Developmental Medicine and Child Neurology, 43(12), 809-818.

Bölte, S., Poustka, F., \& Constantino, J. N. (2008). Assessing autistic traits: cross-cultural validation of the social responsiveness scale (SRS). Autism Research, 1(6), 354-363.

Bruce, B., Thernlund, G., \& Nettelbladt, U. (2006). ADHD and language impairment. European child \& adolescent psychiatry, 15(1), 52-60. 
Cardy, J. E. O., Tannock, R., Johnson, A. M., \& Johnson, C. J. (2010). The contribution of processing impairments to SLI: Insights from attention-deficit/hyperactivity disorder. Journal of Communication Disorders, 43(2), 77-91.

Cohen, N. J., Davine, M., Horodezky, N., Lipsett, L., \& Isaacson, L. (1993). Unsuspected language impairment in psychiatrically disturbed children: Prevalence and language and behavioral characteristics. Journal of the American Academy of Child \& Adolescent Psychiatry, 32(3), 595-603.

Constantino, J. N., Davis, S. A., Todd, R. D., Schindler, M. K., Gross, M. M., Brophy, S. L., \& Reich, W. (2003). Validation of a brief quantitative measure of autistic traits: comparison of the social responsiveness scale with the autism diagnostic interview-revised. Journal of Autism and Developmental Disorders, 33(4), 427-433.

Cuthbert, B. N. (2014). The RDoC framework: facilitating transition from ICD/DSM to dimensional approaches that integrate neuroscience and psychopathology. World Psychiatry, 13(1), 28-35.

Cuthbert, B. N., \& Insel, T. R. (2013). Toward the future of psychiatric diagnosis: the seven pillars of RDoC. BMC medicine, 11(1), 126.

Das, D., Cherbuin, N., Butterworth, P., Anstey, K. J., \& Easteal, S. (2012). A population-based study of attention deficit/hyperactivity disorder symptoms and associated impairment in middle-aged adults. PloS one, 7(2), Article e31500.

DuPaul, G. J., McGoey, K. E., Eckert, T. L., \& VanBrakle, J. (2001). Preschool children with attention-deficit/hyperactivity disorder: impairments in behavioral, social, and school functioning. Journal of the American Academy of Child \& Adolescent Psychiatry, 40(5), 508-515.

Friedman, S. R., Rapport, L. J., Lumley, M., Tzelepis, A., VanVoorhis, A., Stettner, L., ... Kakaati, L. (2003). Aspects of social and emotional competence in adult attention-deficit/hyperactivity disorder. Neuropsychology, 17(1), 50.

Geurts, H. M., \& Embrechts, M. (2008). Language profiles in ASD, SLI, and ADHD. Journal of Autism and Developmental Disorders, $38(10), 1931$.

Granader, Y. E., Bender, H. A., Zemon, V., Rathi, S., Nass, R., \& MacAllister, W. S. (2010). The clinical utility of the social responsiveness scale and social communication questionnaire in tuberous sclerosis complex. Epilepsy \& Behavior, 18(3), 262-266.

Green, B. C., Johnson, K. A., \& Bretherton, L. (2014). Pragmatic language difficulties in children with hyperactivity and attention problems: An integrated review. International Journal of Language \& Communication Disorders, 49(1), 15-29. https://doi.org/10.1111/1460-6984.12056.

Gremillion, M. L., \& Martel, M. M. (2014). Merely misunderstood? Receptive, expressive, and pragmatic language in young children with disruptive behavior disorders. Journal of Clinical Child and Adolescent Psychology, 43(5), 765-776. https://doi.org/10.1080/15374416.2013.822306.

Gut, J., Heckmann, C., Meyer, C. S., Schmid, M., \& Grob, A. (2012). Language skills, mathematical thinking, and achievement motivation in children with ADHD, disruptive behavior disorders, and normal controls. Learning and individual Differences, 22(3), 375-379.

Hagberg, B. S., Miniscalco, C., \& Gillberg, C. (2010). Clinic attenders with autism or attention-deficit/hyperactivity disorder: cognitive profile at school age and its relationship to preschool indicators of language delay. Research in developmental disabilities, 31(1), 1-8.

Hawkins, E., Gathercole, S., Astle, D., Holmes, J., \& Team, C. (2016). Language problems and ADHD symptoms: How specific are the links? Brain sciences, 6(4), 50.

Hutchinson, E., Bavin, E., Efron, D., \& Sciberras, E. (2012). A comparison of working memory profiles in school-aged children with specific language impairment, attention deficit/hyperactivity disorder, comorbid SLI and ADHD and their typically developing peers. Child Neuropsychology, 18(2), $190-207$.

Insel, T., Cuthbert, B., Garvey, M., Heinssen, R., Pine, D. S., Quinn, K., \& Wang, P. (2010). Research domain criteria (RDoC): toward a new classification framework for research on mental disorders. Am Psychiatric Assoc.

Jonsdottir, S., Bouma, A., Sergeant, J. A., \& Scherder, E. J. (2005). The impact of specific language impairment on working memory in children with ADHD combined subtype. Archives of Clinical Neuropsychology, 20(4), 443-456.

Kessler, R. C., Adler, L., Ames, M., Demler, O., Faraone, S., Hiripi, E., \& Spencer, T. (2005). The World Health Organization Adult ADHD Self-Report Scale (ASRS): a short screening scale for use in the general population. Psychological medicine, 35(2), 245-256.

Kessler, R. C., Adler, L. A., Gruber, M. J., Sarawate, C. A., Spencer, T., \& Van Brunt, D. L. (2007). Validity of the World Health Organization Adult ADHD Self-Report Scale (ASRS) Screener in a representative sample of health plan members. International journal of methods in psychiatric research, 16(2), 52-65.

Kim, O. H., \& Kaiser, A. P. (2000). Language characteristics of children with ADHD. Communication Disorders Quarterly, 21(3), 154-165. https://doi.org/10.1177/ 152574010002100304.

Kleinman, A., Caetano, S. C., Brentani, H., Rocca, C. C. d. A., Dos Santos, B., Andrade, E. R., \& Lafer, B. (2015). Attention-based classification pattern, a research domain criteria framework, in youths with bipolar disorder and attention-deficit/hyperactivity disorder. Australian \& New Zealand Journal of Psychiatry, 49(3), 255-265.

Korrel, H., Mueller, K. L., Silk, T., Anderson, V., \& Sciberras, E. (2017). Research Review: Language problems in children with Attention-Deficit Hyperactivity Disorder-A systematic meta-analytic review. Journal of Child Psychology and Psychiatry, 58(6), 640-654. https://doi.org/10.1111/jcpp.12688.

Leonard, M. A., Milich, R., \& Lorch, E. P. (2011). The role of pragmatic language use in mediating the relation between hyperactivity and inattention and social skills problems. Journal of Speech, Language, and Hearing Research, 54(2), 567-579.

Martel, M. M., Von Eye, A., \& Nigg, J. T. (2010). Revisiting the latent structure of ADHD: is there a 'g'factor? Journal of Child Psychology and Psychiatry, 51(8), 905-914.

Martinussen, R., \& Tannock, R. (2006). Working memory impairments in children with attention-deficit hyperactivity disorder with and without comorbid language learning disorders. Journal of Clinical and Experimental Neuropsychology, 28(7), 1073-1094.

McInnes, A., Humphries, T., Hogg-Johnson, S., \& Tannock, R. (2003). Listening comprehension and working memory are impaired in attention-deficit hyperactivity disorder irrespective of language impairment. Journal of Abnormal Child Psychology, 31(4), 427-443.

Mueller, K. L., \& Tomblin, J. B. (2012). Examining the comorbidity of language disorders and ADHD. Topics in Language Disorders, $32(3), 228$.

Munir, K., Biederman, J., \& Knee, D. (1987). Psychiatric comorbidity in patients with attention deficit disorder: A controlled study. Journal of the American Academy of Child \& Adolescent Psychiatry, 26(6), 844-848.

Oram, J., Fine, J., Okamoto, C., \& Tannock, R. (1999). Assessing the language of children with attention deficit hyperactivity disorder. American Journal of SpeechLanguage Pathology, 8(1), 72-80.

Parks, K. M. A., \& Stevenson, R. A. (2018). Auditory and Visual Statistical Learning are not Related to ADHD Symptomatology: Evidence from a Research Domain Criteria (RDoC) Approach. Frontiers in psychology, 9, 2502.

Polanczyk, G., De Lima, M. S., Horta, B. L., Biederman, J., \& Rohde, L. A. (2007). The worldwide prevalence of ADHD: a systematic review and metaregression analysis. American journal of psychiatry, 164(6), 942-948.

Purvis, K. L., \& Tannock, R. (1997). Language abilities in children with attention deficit hyperactivity disorder, reading disabilities, and normal controls. Journal of Abnormal Child Psychology, 25(2), 133-144. https://doi.org/10.1023/A:1025731529006.

Redmond, S. M. (2011). Peer victimization among students with specific language impairment, attention-deficit/hyperactivity disorder, and typical development Language, Speech, and Hearing Services in Schools, 42(4), 520-535.

Redmond, S. M. (2016). Language impairment in the attention-deficit/hyperactivity disorder context. Journal of Speech, Language, and Hearing Research, 59(1), $133-142$.

Redmond, S. M., Ash, A. C., \& Hogan, T. P. (2015). Consequences of co-occurring attention-deficit/hyperactivity disorder on children's language impairments. Language, Speech, and Hearing Services in Schools, 46(2), 68-80.

Russell, J., Jarrold, C., \& Hood, B. (1999). Two intact executive capacities in children with autism: Implications for the core executive dysfunctions in the disorder. Journal of Autism and Developmental Disorders, 29(2), 103-112.

Sciberras, E., Mueller, K. L., Efron, D., Bisset, M., Anderson, V., Schilpzand, E. J., \& Nicholson, J. M. (2014). Language problems in children with ADHD: A communitybased study. Pediatrics, 2013-3355. peds.

Staikova, E., Gomes, H., Tartter, V., McCabe, A., \& Halperin, J. M. (2013). Pragmatic Deficits and Social Impairment in Children with ADHD. Journal of Child Psychology and Psychiatry, 54(12), 1275-1283.

Toplak, M. E., Pitch, A., Flora, D. B., Iwenofu, L., Ghelani, K., Jain, U., ... Tannock, R. (2009). The unity and diversity of inattention and hyperactivity/impulsivity in ADHD: evidence for a general factor with separable dimensions. Journal of Abnormal Child Psychology, 37(8), 1137-1150.

Viscidi, E. W., Johnson, A. L., Spence, S. J., Buka, S. L., Morrow, E. M., \& Triche, E. W. (2014). The association between epilepsy and autism symptoms and maladaptive behaviors in children with autism spectrum disorder. autism, 18(8), 996-1006. 
Wheeler Maedgen, J., \& Carlson, C. L. (2000). Social functioning and emotional regulation in the attention deficit hyperactivity disorder subtypes. Journal of Clinical Child Psychology, 29(1), 30-42.

Wiig, E. H., Semel, E. M., Secord, W., \& Pearson Education, I. (2013). CELF-5: Clinical Evaluation of Language Fundamentals. Bloomington, MN: Pearson.

Wilens, T. E., \& Spencer, T. J. (2010). Understanding attention-deficit/hyperactivity disorder from childhood to adulthood. Postgraduate medicine, 122(5), 97-109.

Yager, J., \& Iarocci, G. (2013). The development of the multidimensional social competence scale: A standardized measure of social competence in autism spectrum disorders. Autism Research, 6(6), 631-641.

http://www.nimh.nih.gov/about/strategic-planning-reports/index.shtml. 2008. (Accessed 15 January 2019). 